Rev Biomed 2001; 12:232-235.

\title{
Manifestaciones cardiacas en pacientes con la infección por el virus de la inmunodeficiencia humana.
} Artículo Original

Renán A. Góngora-Biachi ${ }_{2}^{1}$, Rafael Aguilar-Romero ${ }_{2}^{2}$, Carlos Castro-Sansores ${ }^{1}$, Alejandro GuerreroFlores ${ }^{2}$, Rubén Yza-Villanueva , Armando Fernández-González, Alvaro Quintal-Flores ${ }^{2}$, Carmen Saldivar-Armenta ${ }^{2}$, Pedro González-Martínez .

${ }^{1}$ Centro de Investigaciones Regionales “Dr. Hideyo Noguchi”, Universidad Autónoma de Yucatán, ${ }^{2}$ Centro Médico Nacional “Lic. Ignacio García Tellez”, Instituto Mexicano del Seguro Social, Mérida, Yucatán, México.

\section{RESUMEN.}

Introducción. La afección cardíaca por el virus de la inmunodeficiencia humana ha sido poco descrita. Por lo general es producida por agentes oportunistas. El objetivo de este trabajo fue determinar la prevalencia de afección cardíaca en pacientes con la infección por el VIH.

Material y Métodos. Pacientes con la infección por el VIH de Mérida, Yucatán, México, a los que se les efectuó un exámen clínico, se les completó un cuestionario con datos clínico-epidemiológico y se les realizó electrocardiograma en reposo, teleradiografía de torax y ecocardiograma. Se determinó la prevalencia de afección cardíaca y se compararon las variables categóricas a través de la $X^{2}$ y/o la prueba exacta de Fisher.

Resultados. De junio de 1996 a mayo de 1999, se estudiaron a 37 sujetos, $3(8 \%)$ mujeres y $34(92 \%)$ hombres. Veintisiete $(73 \%)$ se encontraban en etapa Sida y las alteraciones cardiológicas estuvieron presentes en 13 (48\%) pacientes en etapa Sida y en ninguno en otras etapas de la enfermedad ( $\mathrm{p}<0.001)$. Las alteraciones más comúnes fueron la miopericarditis $7 / 13(54 \%)$ sujetos y la miocarditis sola $3 / 13(23 \%)$ pacientes.

Discusión. Se confirma la participación cardíaca en la infección por el VIH, observándose una prevalencia global del $48 \%$. Todos los pacientes que presentaron alteraciones se encontraban en etapa Sida de la enfermedad, lo que confirma que estas alteraciones se presentan en estadios avanzados de la infección.

(Rev Biomed 2001; 12:232-235)

Palabras clave: Sida, VIH, cardiomiopatía.

Solicitud de sobretiros: Dr. Renán A. Góngora-Biachi, Laboratorio de Hematología, Centro de Investigaciones Regionales "Dr. Hideyo Noguchi", Universidad Autónoma de Yucatán, Av. Itzáes No. 490 por 59, C.P. 97000, Mérida, Yucatán, México.

Telefono: (999) 928-41-57, Telecopiadora: (999) 923-61-20. Dirección electrónica: gbiachi@ tunku.uady.mx

Recibido el 25/Febrero/2001. Aceptado para publicación el 9/Mayo/2001.

Este artículo está disponible en http://www.uady.mx/ biomedic/rb011242.pdf

Vol. 12/No. 4/Octubre-Diciembre, 2001 
RA Góngora-Biachi, R Aguilar-Romero, C Castro-Sansores, A Guerrero-Flores y col.

\section{SUMMARY.}

Cardiac manifestations in patients infected with the human immunodeficiency virus.

Background. Cardiac irregularities caused by the human immunodeficiency virus (HIV) have not been described in detail and tend to be subclinical. They are usually produced by opportunist agents. The aim of this study was to determine the prevvalence of cardiac complaints in patients infected by HIV and their relations with the stage of the disease in which they appear.

Methods. Patients infected by HIV in Merida, Yucatan who accepted undergoing a non-invasive cardiaca evaluation were studied. They underwent a clinical examination and completed a questionnaire with clinical-epidemiological data. An electrocardiogram while resting was carried out as well as a radiography of the thorax and a ecocardiogram. The prevalence of cardiac complaints was determined and the categorical variables were compared through the $X^{2}$ and/or Fisher's test.

Results. From June 1996 to May 1999, 37 patients were studied; 3(8\%) women and $34(92 \%)$ men, $27(73 \%)$ were in the AIDS stage of the HIV infection and cardiac irregularities were present in $13(48 \%)$ of these patients, but none in the other stage of the HIV infection $(\mathrm{p}<0.001)$. The most common irregularities were myopericarditis in $7 /$ $13(54 \%)$ patients and myocarditis in $3 / 13$ (23\%) patients.

Conclusions. Our study confirms the cardiac participation in the infection by HIV observing a global prevalence of $48 \%$. All the patients with cardiac irregularities were in the AIDS stage of the disease, which sugest that these cardiac irregularities appear in the advanced stage of HIV infection. (Rev Biomed 2001; 12:232-235)

Key words: Aids, HIV, Cardiomyopathy.

\section{INTRODUCCIÓN.}

La infección por el virus de la inmunodeficiencia humana (VIH) es actualmente uno de los principales problemas de salud en el mundo y sus manifestaciones clínico-patológicas afectan a diversos órganos. Actualmente con el advenimiento de mejores esquemas terapéuticos se ha logrado una mayor supervivencia y el surgimiento de alteraciones clínicas que anteriormente no eran evidentes. Entre éstas, las manifestaciones cardíacas han sido menos documentadas y cuando se han reportado generalmente han sido en estudios postmorten (1) o como notificación de algún caso especial (2), ya que por lo general su curso es subclínico o queda enmascarado por procesos infecciosos oportunistas sistémicos.

La afección cardíaca en los pacientes con el VIH, puede ser producida por la acción de agentes infecciosos oportunistas (3), neoplasias (2) como resultado del tratamiento médico establecido o por acción del VIH mismo (4). Su presentación guarda relación con estados avanzados de la enfermedad y con un estado de inmunosupresión avanzado. Por otra parte, cualquier estructura cardíaca o los vasos sanguíneos puede verse afectada (3).

En este trabajo reportamos la prevalencia y tipo de patología cardiaca en un grupo de pacientes infectados por el VIH en Yucatán, México y su relación con la etapa de la infección por el VIH.

\section{MATERIAL Y MÉTODOS.}

Estudio. Se realizó un estudio prospectivo, transversal y observacional.

Pacientes y Métodos. Pacientes con diagnóstico establecido de la infección por el por el VIH que acudieron por vez primera a valoración médica especializada en el Centro Médico Nacional "Dr. Ignacio García Tellez" del Instituto Mexicano del Seguro Social y al Centro Médico de las Américas en Mérida, Yucatán, México, de junio de 1998 a mayo de 1999 y que como parte del protocolo de estudio les realizaron estudios cardiológicos no invasivos. Los pacientes fueron agrupados en: etapa Sida (Clases A3, B3, C1, C2, y C3) y en etapa no Sida (Clases A1, A2, B1 y B2)

\section{Revista Biomédica}


Cardiopatía asociada al SIDA.

de acuerdo a los criterios del Centro para el Control de la Enfermedades de Atlanta, GA, USA. (CDC) (5). A cada paciente se le realizó: 1) valoración clínica que incluyó un interrogatorio y examen físico y se completó un cuestionario con datos clínico-epidemiológicos; 2) electrocardiograma en reposo; 3) teleradiografía de tórax y 4) ecocardiograma. Para el electrocardiograma se utilizó un electrocardiógrafo marca Zuzuken y el estudio ecocardiográfico se realizó con un Ecocardiógrafo General Electric RT 3000. La realización e interpretación de los estudios electrocardiográficos y ecocardiográficos fue hecha por dos de los autores -médicos cardiólogos- y la conclusión diagnóstica se realizó por consenso entre ellos. De los resultados se determinó la prevalencia de las afecciones cardiacas y se compararon a través de la prueba de la $X^{2}$ y/o la prueba exacta de Fisher.

\section{RESULTADOS.}

Se estudiaron a 37 pacientes infectados por el VIH 3 (8\%) mujeres y 34 (92\%) hombres. La edad promedio del grupo fue 34.8 (4-61) años. Los mecanismos de transmisión de los sujetos adultos fueron: $30(81 \%)$ varones por prácticas homobisexuales y $2(6 \%)$ por prácticas heterosexuales; los 3 casos $(8 \%)$ de mujeres >de 15 años, por prácticas heterosexuales y en $2(6 \%)$ casos pediátricos por transfusión. Ninguno de los pacientes era usuario de drogas intravenosas. Veintisiete $(73 \%$ ) pacientes se encontraban en etapa Sida de la infección, de acuerdo a los criterios establecidos del CDC. Las alteraciones cardiológicas se presentaron en 13/27 (48\%) pacientes en etapa Sida de la infección (12 adultos y 1 niño), y en ninguno de los 10 pacientes en otras etapas de la enfermedad $(\mathrm{p}<0.001)$. La más común fue la miopericarditis que se encontró en $7 / 13$ (54\%) sujetos, la miocarditis sola se halló en 3/13 (23\%) pacientes y se reportaron sendos casos (7.7\%) de mioendocarditis, de pancarditis y de derrame pericárdico. Los diagnósticos fueron sustentados en criterios ecocardiográficos (6) (cuadro 1).

Cuadro 1

Alteraciones cardíacas en 27 pacientes con diagnóstico de Sida.

\begin{tabular}{lcc}
\hline Variable & $\mathrm{n} /$ total & $(\%)$ \\
\hline Alter.Cardíacas: & $\mathbf{1 3 / 2 7}$ & $\mathbf{( 4 8 )}$ \\
Miopericarditis & $7 / 13$ & $(54)$ \\
Miocarditis & $3 / 13$ & $(23)$ \\
Mioendocarditis & $1 / 13$ & $(7.7)$ \\
Pancarditis & $1 / 13$ & $(7.7)$ \\
Derrame pericárdico & $1 / 13$ & $(7.7)$ \\
\hline
\end{tabular}

En 13 pacientes que tuvieron alteraciones cardiológicas (excluyendo un caso de derrame pericáridico) 12 no tenían expresión clínica de ellas. De estos pacientes asintomáticos, 10 (77\%) presentaron también cambios en el electrocardiograma [alteraciones del ST-T (4 casos), bloqueo auriculoventricular (2 casos), taquicardia sinusal (6 casos), trastornos difusos de la repolarización (1 caso)]. En 3 (23\%) pacientes se observaron también alteraciones radiológicas (cardiomegalia en 2 casos y cardiomegalia más hipertensión venocapilar pulmonar en 1 caso). Ninguna de las alteraciones cardiológicas observadas en estos pacientes se relacionó con infecciones oportunistas o enfermedades neoplásicas inherentes a la patología de base de los pacientes.

\section{DISCUSIÓN.}

El primer reporte de afección cardíaca en un paciente con la infección por el VIH se dio en un sujeto haitiano con un sarcoma de Kaposi que afectaba el miocardio y el endocardio del paciente y que se halló en un estudio post-morten (2). Desde entonces se han incrementado el número de estudios que demuestran una mayor participación del corazón dentro del espectro clínico de la infección por el VIH $(7,8)$.

Vol. 12/No. 4/Octubre-Diciembre, 2001 


\section{RA Góngora-Biachi, R Aguilar-Romero, C Castro-Sansores, A Guerrero-Flores y col.}

Nuestro trabajo demostró también la afección cardíaca en el Sida con una prevalencia del $48 \%$, cifra similar a los reportado en otros estudios clinicos en pacientes con Sida (del $36 \%$ al 52\%) $(7,8)$.

La afección pericárdida y la afección miocárdica fueron los hallazgos más frecuentes en concordancia con lo descrito en la literatura $(7,9)$ y la mayoría de los pacientes se encontraban asintomáticos desde el punto de vista cardiológico y el hallazgo diagnóstico fue a través de ecocardiografía. Este hecho apoya la utilidad del ecocardiograma como la mejor herramienta diagnóstica de las manifestaciones cardíacas en el Sida, ya que el electrocardiograma y la teleradiografía de tórax fueron menos útiles y menos sensibles para la detección de éstas.

Aunque la endocarditis ha sido descrita como una afección frecuente en los enfermos con Sida, en este estudio la afección endocárdica tuvo poca participación. Este hecho seguramente se debió a que esta patología se asocia de manera importante al uso de drogas intravenosas (10) que en este grupo de enfermos no estuvo presente. Todos los pacientes que presentaron alteraciones cardíacas se encontraban en etapa Sida de la infección, lo que confirma que éstas alteraciones se presentan en estadios avanzados de la enfermedad.

Se concluye que las manifestaciones cardíacas en el Sida son más frecuentes que lo que clínicamente se puede detectar, ya que cursan la mayoría de las veces en forma desapercibida o enmascarada por la sintomatología predominante de este síndrome. Por esto se debe incluir estudios cardiológicos dentro de la evaluación integral del paciente con Sida, para poder hacer una detección temprana y así implementar las medidas terapéuticas precisas.

\section{REFERENCIAS.}

1.- Cammarosano C, Lewis W. Cardiac lesions in acquired immunodeficiency syndrome (AIDS). J Am Col Cardiol 1985; 5: 703-6.

2.- Autran BR, Gorin 1, Leibowitch M. AIDS in Haitian woman with cardiac Kaposi's sarcoma and Whipple's disease. Lancet 1983; 1: 767-8.

3.- Yunis NA, Stone VE. Cardiac Manifestations of HIV/ AIDS. J Acquir Immune Defic Syndr 1998; 18: 145-54.

4.- Lipshultz SE, Fox CM, Pérez-Atayde AR. Identification of human immunodeficiency virus-1 RNA and DNA in the heart of a child with cardiovascular abnormalities and congenital acquired immunodeficiency syndrome. Am J Cardiol 1990; 66: 246-51.

5.- Center for Diseases Control. 1993 revised classification system for HIV infection and expanded survillance case definition for AIDS among adolecents and adults. MMWR 1993; 41: 1-19.

6.- Hammoude A. Endocardial border identification in twodimensional echocardiographic images. Review of methods. Comput Med Imaging Graph 1998; 22: 181-193.

7.- Monzues JJ, Kinney EL, Vittecoq D. Comparison among acquired immuno deficiency syndrome patients with and without clinical evidence of cardiac disease. J Am Coll Cardiol 1988; 12: 1211-13

8.- Anderson DW, Virmani R, Reilly JM, O’Leary TJ. Prevalent myocarditis at necropsy in acquired immunodeficiency syndrome. J Am Coll Cardiol 1988; 11: 792-99.

9.- Reilly JM, Cunnion RE, Anderson DW, O’Leary TJ, Simmons JT, Lane HC. Frequency of myocarditis, left ventricular dysfunction and ventricular tachycardia in the acquired immune deficiency syndrome. Am J Cardiol 1988; 62: 789-93.

10.- Stoneburger RL, Des Jarlais DC, Benezra D. A larger spectrum of severe HIV-1 related disease intravenous drug users in New York City. Science 1988; 242: 916-9. 